Review

Saskia G. von Diest

\title{
Could biodynamics help bridge the gap in developing farmer intuition?
}

https://doi.org/10.1515/opag-2019-0036

received August 30, 2018; accepted May 23, 2019
Keywords: Intuition; Tacit knowledge; Biodynamic agriculture; Experiential learning; Ecofluency

\section{Introduction}

Until recently, biodynamics has not featured heavily in research or publications coming out of mainstream agricultural science because of its perceived esoteric nature. However, the increasing number of biodynamic farmers in a growing number of countries has led to renewed interest in biodynamic farming from the scientific community (Code 2018).

This article has three aims: The first is to identify a theoretical and practical knowledge gap in modern agricultural science where farmer decision-making is concerned. The second aim is to explore how that gap could be narrowed through collaborating with the theory and practice of biodynamics. The third aim is to describe what might be the potential benefits of such collaboration for biodynamics, mainstream agricultural science and farmers in general.

\subsection{The knowledge gap in modern science on farmer decision-making}

Farm management decision-making has been greatly advanced through modern (Newtonian-Cartesian) science. This can be attributed to the focus of modern science on the generation of explicit knowledge using a mainly logical approach. Explicit, or formal, knowledge can be documented, codified, articulated and easily transferred in a systematic and tangible form using numbers, formulae, words and manuals (Boateng 2006; Nonaka and van Krogh 2009; Vangala et al. 2014).

This rational approach has accelerated the development of technological tools in the last few decades, such as information communication technology and precision agriculture, that aim to support farmer decision-making (Fountas et al. 2006). These formalised tools are based 
on cognitive task analysis, and are generally designed to bridge the knowledge gap between agricultural science and farming practices to streamline farm management decisions. While such tools have been adopted and are useful to many farmers when making difficult decisions (Bramley 2009; Fountas et al. 2015), several studies have found that farmers often do not adopt formalised tools as expected, and prefer making use of other internal decision-making skills, such as intuition (Lynch et al. 2000; Alvarez and Nuthall 2006; Öhlmér 2007; McCown 2012; Kieft 2015; Nuthall and Old 2018).

It appears that the development of such tools is rarely done with consideration for, and a detailed understanding of, the relationship between the specific knowledge that farmers have, and the way in which farmers arrive at the decisions they make (Alvarez and Nuthall 2006; Öhlmér 2007; Robert et al. 2016).

Additionally, formalised tools are only available to those farmers who have access to them and who understand how to use them, which is rarely the case for indigenous, small-scale, smallholder and subsistence farmers, and especially in less industrialised countries. These farmers use their tacit understanding to employ practices to adapt to increasingly unpredictable climatic conditions, such as drought (Kieft 2006, 2015; IAASTD 2009), indicating the value of tacit knowledge in developing resilience of farming systems. Their situational knowledge (Haraway 1988), generated in situ through experience, language, culture, tradition and savoire-faire (the adaptive ability to determine appropriate action), is often the product of tacit learning. Tacit, or intangible, personal knowledge, is informal and implicit in nature, involving perspective, personal beliefs and values, and it cannot always be codified, articulated, or transferred (Nonaka 1994; Sinclair and Ashkanasy 2005; Boateng 2006; Nonaka and van Krogh 2009).

Tacit knowledge is, of course, not limited to indigenous, small-scale and subsistence farmers. However, the importance of farmers' tacit knowledge has not yet filtered significantly into mainstream agricultural research, and it is rarely discussed in large-scale or industrialised agricultural sectors that rely on modern science, which places emphasis on explicit knowledge. This indicates a disparity between modern science's current knowledge of farmer decision-making and the kinds of knowledge, like tacit knowledge, to which farmers have access and on which they rely, which are not purely rational.

So how then can modern science be used to better support farmers and diverse farming practices? If modern science is currently limited in its ability to access - let alone appropriately operationalise - these different kinds of knowledge, then perhaps a more prudent approach is to find ways to support farmers in developing their own field of (multisensory) awareness and ability to effectively access and utilise tacit knowledge.

\subsection{What is understood by intuition?}

Intuition, or 'knowing from within', has been described as one method of accessing tacit knowledge (Sinclair and Ashkanasy 2005; Boateng 2006; Nonaka and van Krogh 2009). Intuition is a sub-conscious part of all decisions and plays a role in creative decision-making. It allows extra, valuable information to be acquired, beyond what is already present using logical cognitive processes and the conscious mind. It also offers the advantage of being complete, quick, accurate and confidence boosting, especially when there is a time constraint, or in new or complex situations (Claxton 1998; Khatri and Ng 2000; Hodgkinson et al. 2008; Lufityanto et al. 2016). In business management, intuition is considered a pervasive, involuntary and automatic approach that managers can harness and use in situations when they are able to recognize and manage it (Sadler-Smith and Shefy 2004).

Jung (1976 [1921]) outlines four basic functions of the conscious human psyche that contribute equally to adaptation and orientation: sensation (perception through sense organs), thinking (intellectual cognition and logical judgement), feeling (subjective valuation) and intuition. He describes intuition as necessary for holistic thinking, defining it as "perception by way of the unconscious, or perception of unconscious contents" that enables humans to "divine hidden possibilities in the background".

About two decades before Jung, Rudolf Steiner, the founder of anthroposophy and biodynamic agriculture, considered intuition as the highest stage of non-physical perception, a superconscious stage of knowledge, and as pivotal to examining one's own thoughts in the quest for self-awareness (Cain 2016). In addition to his own insights, Steiner was inspired by many ancient philosophical traditions and their texts, such as the Vedanta in ancient India. This may have influenced his unique perspective for his time of how, not just human-related, but other tangible and intangible factors, influence human decision-making and behaviour.

Steiner made it clear that the maps he was attempting to draw to navigate the landscape of the human psyche were not the landscape itself, and was careful in articulating what he meant. Because of the lack of appropriate language available for translating experiences in the non-verbal, super-sensible worlds (beyond what can be 
experienced through the five physical senses) to physical worlds, he deliberately pointed out that he often only hinted at what he felt would be hubris to put into words (Steiner 1995 [1894]; vii).

There is currently no consensus on what is meant by intuition, even by farmers who state that they rely on their intuition (Paxton et al. 2017). If used sub-consciously, it could be considered a latent resource, whereas when used consciously to any degree, it is a resource that could benefit from refinement. Nevertheless, it is a potentially highly useful faculty present in all farmers worldwide, and the use and development of it deserves more attention in farmer decision-making research (Paxton et al. 2017; Nuthall and Old 2018). Doing so could offer a means of empowering farmers to individually access and apply tacit knowledge that may be more appropriate for their own circumstances (Boateng 2006).

\subsection{An alternative to externalising tacit knowledge to fill the knowledge gap}

Studies that have focused on farmers' tacit knowledge have done so with the aim of improving and developing regenerative agricultural practices through externalising and converting tacit knowledge into explicit knowledge (van Eijk 1998; Boateng 2006; Curry and Kirwan 2014; Vangala et al. 2014; Semeon et al. 2015). There is undoubtedly great value in obtaining such information, but there may be a risk that, because of the internal, experiential nature of tacit knowledge that is situational and embedded in particular cultures, the practices developed from them might not be suited to adaptation to, or scaling up in, other cultures and regions (Chilisa 2012).

Since tacit knowledge cannot easily be articulated and transferred, could such an externalisation process alter or dilute the value of this form of knowledge? Furthermore, given that ICT tools are often not adopted or used as expected, if such information were incorporated into ICT tools, would these tools be accessed and adopted by farmers? According to Hochman and Carberry (2011), support systems should help farmers develop their intuition instead of replacing it with optimised recommendations.

The same studies that found that farmers did not adopt formalised tools as expected also found that many farmers prefer an intuitive approach to an analytic system (Lynch et al. 2000; Öhlmér 2007; McCown 2012; Kieft 2015; Nuthall and Old 2018). As described above, the design of formalised tools rarely, if ever, considers the other forms of specific knowledge that farmers have and use, or how they actually make decisions. As Öhlmér (2007) puts it, "We know what farmers should do, but not so much about what farmers actually are doing".

So, if some farmers prefer relying on intuition, what are the benefits of doing so? In a study by Paxton et al. (2017) of 79 organic farmers in Germany, Austria and the UK, the development of intuition was one of ten key points agreed to be vital for the running of healthy farming systems. As one farmer in the study put it: "We're talking about things that are not actually tangible... something older, something that we have lost, like intuition". Other farmers in the study stated that farm health management needs to account for the individuality of the farm, and not just use blueprint solutions, and that intuition allows customised practical decisions.

Farmers surveyed in the Netherlands, Peru, Brazil and Sri Lanka also reported that relying primarily on their intuition allowed them to secure considerable benefits, such as lower inputs (e.g. water, fertilizer, vet costs, time used for making decisions), improved outputs (e.g. feed conversion in animal production, longer product shelf-life, higher yield), greater profit margins, improved farm health and reduced negative environmental impact, improved personal well-being (e.g. feeling healthier, more satisfied with their decisions and more in harmony with nature) (Kieft 2006, 2015).

Regenerative farming requires more locally appropriate strategies and practices (Pimbert 2015), which implies more localised decision-making, i.e. decision-making tailored to a farm's specific set of conditions and requirements, which then falls to the responsibility of the farmers. If so, then perhaps it would make more sense if farmers were to be supported in developing intuition, that could complement their analytic processes, and allow them to make quick, customised, decisions that are suitable to their particular situation (Nuthall 2012; Kieft 2015; Nuthall and Old 2018).

\subsection{How could farmer intuition be developed?}

As intuition is an innate part of all decisions, focusing on it could offer farmers a potentially valuable skill to support and complement their decision-making. Focusing research attention on farmer intuition does not suggest that farmers should use explicit knowledge or their analytical, logical abilities any less, nor does it suggest that research into tools that support their analytic abilities should not continue. However, assuming that farmers would need and/or want support from research focusing on intuition 
development, this might imbue them with more agency, autonomy and responsibility than approaches that make them further reliant on external sources of advice.

The true success rate of intuition is unknown, and while many authors describe it as accurate (Claxton 1998; Khatri and Ng 2000; Hodgkinson et al. 2008; Lufityanto et al. 2016), it can still be fallible. One of the biggest challenges may be overcoming implicit bias, and specifically confirmation bias, to find results and knowledge that confirm one's method of decision-making. However, openminded questioning and reflexivity can help sharpen discernment. Training increases the reliability of intuitive decision-making: just as people become more comfortable when applying logic and reasoning in making decisions, people can also become more adept at trusting their intuition when used more frequently (Lufityanto et al. 2016). Many experts hone their intuition over years of job-specific experience (Khatri and Ng 2000; Nuthall 2012) and farmers agree that intuition can be learned (Paxton et al. 2017; Nuthall and Old 2018).

So far, only one published study in the agricultural sector by Nuthall and Old (2018) has addressed the question of how farmers can develop their intuition for management decision-making, with the aim to develop an original model of explaining intuition and how it may be developed based on the variables that the authors have found to influence intuitive decision-making. While the insight gained through studies like these would be highly valuable, there is a risk in treating intangible knowledge in the same manner as explicit knowledge. As discussed earlier, such approaches to tacit knowledge and subtle abilities could mean that progress remains limited because of the generally reductionist nature of the scientific approach, in which dynamic, living processes, both external and internal, are reduced to the parts of a process or system for study. Intuition is not easily verbalized (Hodgkinson et al. 2008), and so methods used to train analytical skills may not be entirely appropriate for developing subtle abilities such as intuition.

Since tacit knowledge can be accessed through tactile experiences, movement skills and reflection (Nonaka and van Krogh 2009), embodied practices and experiential learning may offer an additional approach to refining intuition development that may be more appropriate.

While intuition is not emotional, biased, or opposed to rationality (Khatri and $\mathrm{Ng}$ 2000), intuitive decisions do seem to be influenced by subtle emotional priming (Goodyear et al. 2016), suggesting that recognition and management of one's emotional triggers may be an important part of developing intuition (Kahneman 2003; Sinclair et al. 2010).
People vary in their intuitive abilities, but self-critique, structured reflection and reflexive processes are crucial to developing confident and informed intuition (Nuthall and Old 2018). According to farmers who claim to use their intuition in managing farm health, personal development is closely tied to building the intuitive capacity, with self-observation and self-reflection needed for farmers to match their abilities with the complexity of their farm (Paxton et al. 2017). This aspect of developing intuition places responsibility on the farmer to actively engage in personal development, which is likely to be more challenging than sitting in a course or workshop and passively absorbing explicit knowledge. However, consultation with friends and colleagues, which is also an important part of intuition development (Nuthall and Old 2018) may offer farmers support in this process.

So, if intuition development might be facilitated with embodied experience, and requires engaging in reflective and reflexive processes, this presents an opportunity for developing new research methodologies for the scientific approach.

One of the most appropriate approaches currently may be emergent transdisciplinary design research (ETDR), in which the research design emerges as the research process unfolds (Rosenberg 2017; van Breda and Swilling 2018). This has evolved from transdisciplinary, participatory methods, which position the researchers as co-inquirer(s) in a reciprocal relationship with study participants, rather than subjects (Lang et al. 2012; Chilisa 2012: 189194; Madjidi 2014). These approaches favour an evolution of endogenous (generated from within) knowledge over an extractive approach to knowledge production.

Such research deliberately requires both acknowledgement and inclusion of both the researcher's and study participant's beliefs and values during the research process, as well as the involvement of the researcher's own intuition in making key decisions in developing the research process (van Eijk 1998; Rosenberg 2017). It also requires that the researcher be open and willing to potentially undergo a personal transformation, particularly of the filters of the lenses with which they view reality.

\subsection{How could biodynamics help farmers develop intuition?}

While biodynamic certification only regulates physical practices on farms, the teachings of Steiner encourage farmers to develop their self-observation abilities and incorporate the information gained in their practical decision processes, as will be described below. Academic 
data is not easily available on the extent to which biodynamic farmers use these practices. However, in the study by Paxton et al. (2017), biodynamic farmers are more comfortable with speaking about their feelings and the concept of intuition than organic farmers (Anja Vieweger personal communication). Based on the website of a biodynamic farm in Kentucky, USA, "biodynamic agriculture emphasizes the importance of the farmer's personal relationship with the land. Keen observation... and following one's intuition are vital to the success and quality of biodynamic farming" (https://foxhollow.com/what-is-biodynamics). Similarly, a farmer in Ontario, Canada, who converted from conventional to biodynamic farming stated that "Observation and intuition were guiding lights for communities whose members worked together to feed themselves" (https://www.producer.com/2016/02/agriculture-at-the-crossroads/).

That there is a systematic approach available to farmers means that biodynamic agriculture is the only form of modern, Western alternative agriculture that proactively recognizes and encourages intuitive farming.

As discussed above, personal development seems to be an important part of coming to grips with intuitive ways of knowing. Steiner's perspective on intuition could be helpful in addressing the knowledge gap in modern science about intuition and intuition development, both within and beyond agriculture.

Steiner was careful to articulate his understanding of intuition, and provided systematic guidelines for honing the 'subtle' abilities of humans, in a similar way to how the intellect can be schooled: In what is often described as the most fundamental book that Steiner wrote, 'The Philosophy of Freedom' (1894), he describes what he learned when applying observational methods of natural science to super-sensible worlds, such as Goethean observation.

In 'Knowledge of the Higher Worlds and its Attainment' (translated), Steiner outlines practices for the reader to develop super-sensible perception - "When rightly applied in life, knowledge of the super-sensible worlds proves not to be impractical, but practical in the highest degree" (Steiner 2012 [1904]; 5). In a subsequent publication, 'The Stages of Higher Knowledge', he describes imagination, inspiration and intuition as three human faculties that are dynamically and consecutively part of the development of super-sensible perception. He describes intuition as a non-dual experience of the other, in terms of there being no subject or object relationship between the perceiver and the perceived. In this work, he states "Intuition is not a mode of cognition which with regards to clarity lags behind intellectual knowledge, but one that far surpasses it” (Steiner 1967 [1905]). This agrees with authors in modern science who describe the accuracy and immediacy of intuition (Claxton 1998; Khatri and Ng 2000; Hodgkinson et al. 2008; Lufityanto et al. 2016).

Steiner's works appear to be the only set of systematic guidelines for developing not only super-sensible perception and intuition, but also what I more broadly define as 'ecofluency': the ability to fluidly and accurately converse with more-than-human nature using the expanded spectrum of human sensory awareness.

Etymologically, 'eco' is derived from 'oikos' (Greek) meaning house or habitation, and 'fluency' from 'fluentem' (Latin) meaning free-flowing or relaxed. This builds on ecoliteracy, which applies a more holistic understanding of complex systems thinking in order to 'read', or sense, into "the book of nature" (Code 2019). Yet, as outlined by Code, 'ecoliteracy' is limited by both one's definition of 'book' and what it actually means to be 'literate': literacy tends to be dependent on explicit knowledge and one's ability to effectively comprehend and interpret it.

Additionally, perceiving nature as a book implies that there is a passive uni-directional 'reading' of 'nature'. Ecofluency, as I propose, goes a step beyond: it not only requires fostering an intimate comprehension of the functioning of interconnected ecological systems, but it invites us to acknowledge and apply our understanding of the bi-directionality of our participation with/in 'nature', to cultivate a sense of harmony (flow) through a process of co-creation (dialogue) with the more-than-human world.

This requires an expanded perspective of 'nature': one that shifts from seeing it as a background for human activity and food growing, to an animate web of inter-related organisms with which we can commune through a range of intuitive approaches. Indeed, the ability to integrate multiple perspectives on the human-nature relationship is considered vital in progressing the many fields that comprise the scientific study of ecology (Zylstra et al. 2018), and, therefore, agro-ecology. Integrating the rational and intuitive faculties of human awareness allows one to develop 'a feel' or 'attunement' for the more-than-human world and, in time, bring one into closer relation with biodiversity - an approach deemed vital for averting the 'extinction of experience' (cf. Samways 2007).

The practice of ecofluency also encourages more regular, direct and meaningful contact with nature and the sublime - elements that inculcate a sense of connectedness and, in turn, fosters a conservation ethic that supports pro-environmental behaviour (Pyle 2003; Saunders 2003; Miller 2005; Zylstra et al. 2014, 2018). This might help meet the need for farmers to match their own abilities with the unique complexity of their farm, as described by intuitive farmers in the study by Paxton et al. (2017). 
As has been described by Samways (2007) and Zylstra et al. (2018), the primacy of direct experience needs to be emphasized if the collective human relationship with nature, upon which our food systems are also based, is to foster greater connectedness and harmony and, through that, transform the food systems themselves. Steiner, in his Agriculture Course lectures, does this by inviting farmers to act accordingly in their function as an organ in their farm's 'self-contained individuality', as he refers to self-sufficient agroecosystems. Intuition, as described in Steiner's texts above, could be a step in developing intuitive communication with nature, or ecofluency, and the necessary evolution of the human-nature relationship.

Jung and other authors describe intuition as an unconscious function. Steiner considers intuition a superconscious stage of knowledge that can be developed or attained through particular, yet customisable, methods that include his theory about the origins of intuition, and places the greater part of the responsibility in the hands of the individual. Therefore, Steiner's perspective on the value and development of intuition may be a comprehensive, or holistic, approach that could be adopted by different individual farmers, groups of farmers and cultures to suit and complement their particular needs in developing their intuition and other subtle abilities.

Biodynamics already has a global presence, practiced in at least 47 countries with a growing number of associations and members. This means that there is an existing network, and through which researchers could contact biodynamic farmers. It also provides easier access for both biodynamic and non-biodynamic farmers to Steiner's teachings, and to other individuals and groups who may share similar goals in developing their intuition and other subtle abilities. This may be important, because, as mentioned earlier, intuition development is supported by consultation with friends and peers.

For a long time, biodynamic philosophy and practice in and of the non-material world has been its most underplayed characteristic. But it may offer the rest of the farming community valuable inspiration and insight, and a more open conversation between biodynamics and the rest of the agricultural world needs to be had.

\subsection{Potential benefits of collaborative research}

If the knowledge gap could be, at least partially, addressed through collaborative explorations between modern agri- cultural science and biodynamic theory and practice, then there may be a number of benefits to both parties.

Because of the seemingly mysterious nature of biodynamics, it has often been ignored, and even dismissed, by mainstream researchers and conventional farmers. The biodynamic community could benefit from scientific findings that recognise the value of biodynamic practices. This could help to provide a bridge between biodynamic and conventional farmers, which might help change how conventional farmers perceive biodynamic agriculture. This may help spread the awareness and practice of biodynamics. It may also encourage biodynamic farmers to deepen their intuitive abilities and more consciously use them in practical decision-making.

There may be similarities between what is taught in Steiner's work about intuition and what has been learned through modern science. For example, Steiner encourages those who wish to develop their super-sensible abilities, such as intuition, to become more aware of the sensations that arise within the body. Electrophysiological evidence has found that the heart is involved in the reception, processing, and decoding of intuition (McCraty et al. 2004), and it appears that the relationship between intuition and visceral sensations, or 'gut feelings', may share a common, perceptive origin (Radin and Schlitz 2005). Both of these perspectives support the use of embodied and experiential learning in training intuition.

There is already a basis in modern science of theories and methodologies for facilitation and support of individual and group processes, towards personal and collective transformation, such as the studies by Madjidi (2014), Zylstra (2014) and van Eijk (2015). If successful, the outcomes of collaboration between agricultural science and biodynamics could add to and help develop this knowledge base.

New research methodologies for such collaboration may need to be developed, which may also require a development or expansion of the lexicon available to researchers and study participants, or co-inquirers. Ecofluency may be one such term that could be added to this lexicon, in helping to overcome 'linguistic or scientific imperialism' - the notion that so much of the externalised land management guidance is produced in English and/ or with a scientific worldview, which may be difficult for farmers to understand, especially small-scale and subsistence farmers. Encouraging intuition, and ecofluency, might go some way towards bypassing those roadblocks, and there is much to be learned from the language used in anthroposophy and biodynamic farming. Anthroposophical language is more creative and abstract, and may be more appealing to farmers when engaging intuition 
development than the kind of language used to generate and impart explicit knowledge. This may be particularly useful when working with indigenous and traditional farmers, whose worldview of recognising the importance of interacting with the non-material world may resonate more with the anthroposophical approach that informs biodynamics, than that of the materialist worldview that is dominant in modern science.

\section{Conclusion}

In this exploratory article, a theoretical and practical gap in knowledge in modern agricultural science on farmer decision-making was identified, and reasons given for why that gap may need to be addressed with non-conventional scientific approaches. A proposal is made here as to how the teachings behind biodynamics may help to bridge that gap, and the potential benefits of an alliance between biodynamics and mainstream agricultural science and farmers are explored.

Supporting farmers in refining their intuitive abilities would be a practical and strategic approach for helping farmers to access tacit knowledge and make quick, accurate, customised decisions in systems that are complex and dynamic. This could complement farmers' explicit knowledge and analytical abilities, and would be especially useful for farmers who do not have access to the technology upon which formalised decision support tools rely. A forthcoming article will explore the literature on that particular topic in more detail. Furthermore, this might be supportive for farmers, and even non-farmers, in regaining agency for, and confidence in, their own inner knowing, or 'knowledge compass', and belief that they can have the ability to access the relevant information they need at a given time.

Based on the arguments made here, it appears that collaborative explorations between modern agricultural science and biodynamic theory and practice could provide an innovative and more adequate approach to investigating the development of farmer intuition than a purely analytical one, and so potentially begin to close the knowledge gap. Steiner's works offer a different type of language that could help expand the lexicon for such research, add to the understanding and body of literature on intuition, and provide a basis for ecofluency.

Such an alliance may help to reinvigorate agricultural research towards more efficient, customised and coherent practices and, ultimately, resilient farming systems.
Acknowledgements: E. Cloete, Vice-Rector of Innovation and Research, Stellenbosch University, for support and discretionary funding, as well as J. Wright, J. Code and M. Zylstra for valuable commentary.

This work was supported by funding from the Department of Science and Technology (grant number 81789/2012) and the National Research Foundation (grant number 95435) of South Africa, as well as by funding from Coventry University, UK.

\section{References}

[1] Alvarez J., Nuthall P., Adoption of computer based information systems: The case of dairy farmers in Canterbury, NZ, and Florida, Uruguay, Comput. Electron. Agric., 2006, 50, 48-60

[2] Boateng W., Knowledge management working tool for agricultural extension: the case of Ghana, Knowl. Manag. Development J., 2006, 2, 19-29

[3] Bramley, R.G.V., Lessons from nearly 20 years of Precision Agriculture research, development, and adoption as a guide to its appropriate application. Crop Pasture Sci., 2009, 60, 197-217

[4] Cain J.L., The Aesthetics of Rudolf Steiner and Spiritual Modernism. PhD dissertation, University of Michigan, Ann Arbor, Michigan, USA, 2016

[5] Chilisa B., Indigenous Research Methodologies. Sage Publications, Los Angeles, 2012

[6] Code J., Alternative agriculture: Innovations for growing and cultivating diverse ways of knowing, In: Zeunert J., Waterman T. (Eds.), Routledge Handbook of Landscape and Food, Routledge, London, 2018

[7] Code, J., Ecoliteracy and the trouble with reading: ecoliteracy considered in terms of Goethe's 'delicate empiricism' and the potential for reading in the book of nature. Environ. Educ. Res., 2019, https://doi.org/10.1080/13504622.2018.1558438

[8] Curry N., Kirwan J., The role of tacit knowledge in developing networks for sustainable agriculture. Sociol. Ruralis, 2014, 54, 341-361

[9] Fountas S., Carli G., Sørensen C.G., Tsiropoulos Z., Cavalaris C., Vatsanidou A., Farm management information systems: Current situation and future perspectives Comput. Electron. Agric., 2015, 115, 40-50

[10] Fountas S., Wulfsohn D., Blackmore B.S., Jacobsen H.L., Pedersen S.M., A model of decision-making and information flows for information-intensive agriculture, Agric. Sys., 2006, 87, 192-210

[11] Haraway D., Situated Knowledges: The Science Question in Feminism and the Privilege of Partial Perspective, Fem. Stud., 1988, 14, 575-599

[12] Hochman Z., Carberry P.S., Emerging consensus on desirable characteristics of tools to support farmers' management of climate risk in Australia, Agric. Sys., 2011, 104, 441-450

[13] Hodgkinson G.P., Langan-Fox J., Sadler-Smith E., Intuition: A fundamental bridging construct in the behavioural sciences, Brit. J. Psychol., 2008, 99, 1-27 
[14] IAASTD (International Assessment of Agricultural Knowledge, Science and Technology for Development), Agriculture at a crossroads: A synthesis of the global and sub-global IAASTD reports. McIntyre, B.D., Herren, H.R., Wakhungu, J., Watson, R.T. (Eds.) Island Press, Washington D.C., USA, 2009

[15] Jung C.G., Psychological Types, Princeton University Press, New York, USA, 1976

[16] Kahneman, D., Maps of Bounded Rationality: Psychology for Behavioral Economics. Am. Econ. Rev., 2003, 93, 1449-1475

[17] Khatri N., Ng H. A., The role of intuition in strategic decision making, Hum. Relat., 2000, 53, 57-86

[18] Kieft H., Intuitive Farming: Towards a New Vision on Nature. Proceedings of the XI International People-Plant Symposium on Diversity: Towards a New Vision on Nature, Acta Hort., 2015, 1093, 179-194

[19] Kieft H., Quantum Agriculture: bridging frontline physics and intuitive knowledge of nature? In: Haverkort B., Reijntjes, C. (Eds)., Moving Worldviews, reshaping sciences, policies and practices for endogenous sustainable development. ETC/ COMPAS, Leusden, The Netherlands, 2006, 209-218

[20] Knowler D., Bradshaw B., Farmers' Adoption of Conservation Agriculture: A Review and Synthesis of Recent Research, Food Pol., 2007, 32, 25-48

[21] Lang D.J., Wiek A., Bergmann M., Stauffacher M., Martens P., Moll P., et al., Transdisciplinary research in sustainability science - practice, principles and challenges, Sustain. Sci., 2012, 7 (Supplement 1), 25-43

[22] Lufityanto G., Donkin C., Pearson J., Measuring Intuition: Nonconscious Emotional Information Boosts Decision Accuracy and Confidence, Psychol. Sci. 2016, 27, 622-634

[23] Lynch T., Gregor S., Midmore D., Intelligent support systems in agriculture: how can we do better? Aust. J. Exp. Agric., 2000, 40, 609-620

[24] McCown R.L., A cognitive systems framework to inform delivery of analytic support for farmers' intuitive management under seasonal climatic variability, Agric. Sys., 2012, 105, 7-20

[25] McCown R.L., Carberry P.S., Dalgliesh N.P., Foale M.A., Hochman Z., Farmers use intuition to reinvent analytic decision support for managing seasonal climatic variability, Agric. Sys., 2012, 106, 33-45

[26] McCraty R., Atkinson M., Bradley R.T., Journal of Alternative and Complementary Medicine Electrophysiological Evidence of Intuition. Part 1, the Surprising Role of the Heart. Part 2, a System Wide Process. J. Altern. Complement. Med., 2004, 10, 133-143 and 325-336

[27] Miller J.R., Biodiversity conservation and the extinction of experience. Trends Ecol. Evol., 2005, 20, 430-434

[28] Nonaka I., A dynamic theory of organizational knowledge creation, Organ. Sci., 1994, 5, 14-36

[29] Nonaka I., van Krogh, G., Tacit Knowledge and Knowledge Conversion: Controversy and Advancement in Organizational Knowledge Creation Theory, Organ. Sci., 2009, 20, 635-652

[30] Nuthall P.L., Old K.M., Intuition, the farmers' primary decision process: A review and analysis, J. Rural Stud., 2018, 58, 28-38

[31] Nuthall P.L., The intuitive world of farmers - The case of grazing management systems and experts, Agric. Sys., 2012, 107, 65-73

[32] Öhlmér B., The Need and Design of Computerized Farm Management Tools - Lessons Learned from a Swedish Case,
Working Paper Series 2007: 5, Department of Economics, SLU, Uppsala, 2007

[33] Paxton R., Klimek M., Vieweger A., Döring R., Bloch R., Bachinger J., Woodward L., "The Role of Intuition in Managing Organic Farm System Health” in: Rahmann, Gerold (Ed.) et al.: Innovative research for Organic 3.0 - Volume 1: Proceedings of the scientific track at the Organic World Congress 2017, November 9-11 in Delhi, India. Thünen Report, 2017, No. 54,1

[34] Pimbert M., Agro-ecology as an Alternative Vision to Conventional Development and Climate-smart Agriculture, Development, 2015, 58, 286-298

[35] Pyle RM., Nature matrix: reconnecting people and nature, Oryx, 2003, 37, 206-214

[36] Radin D., Schlitz M.J., Gut Feelings, Intuition, and Emotions: An Exploratory Study, J. Altern. Complement. Med., 2005, 11, $1,85-91$

[37] Robert M., Dury J., Thomas A., Therond O., Sekhar M., Badiger S., Ruiz L., Bergez J-E., CMFDM: A methodology to guide the design of a conceptual model of farmers' decision-making processes, Agric. Sys., 2016, 148, 86-94

[38] Rosenberg L.L., Turi kumwe (we are together): A transdisciplinary exploration of the Burundian specialty coffee sector and its sustainability challenges, PhD Dissertation, Stellenbosch University, Stellenbosch, South Africa, 2017

[39] Sadler-Smith E., Shefy E., The intuitive executive: Understanding and applying 'gut feel' in decision-making, Acad. Manag. Learn. Educ., 2004, 18, 76-91

[40] Samways, M., Rescuing the extinction of experience. Biodivers. Conserv., 2007, 16, 1995-1997

[41] Saunders C.D., The emerging field of conservation psychology. Hum. Ecol. Rev., 2003, 10, 137-149

[42] Sinclair, M., Ashkanasy, N. M., Chattopadhyay, P., Affective antecedents of intuitive decision making. J. Manag. Organ., 2010, 16, 382-398

[43] Sinclair, M., Ashkanasy, N. M., Intuition: Myth or a decision-making tool? Manag. Learn., 2005, 36, 353-370

[44] Steiner R., Intuitive Thinking as a Spiritual Path; A Philosophy of Freedom. Centennial Edition, Anthroposophic Press, Hudson, New York, USA, 1995

[45] Steiner R., Knowledge of the Higher Worlds and How it is Achieved, Rudolf Steiner Press, Forest Row, UK, 2012

[46] Steiner R., The Stages of Higher Knowledge, Anthroposophic Press, Hudson, New York, USA, 1967

[47] Van Breda J., Swilling M., The guiding logics and principles for designing emergent transdisciplinary research processes: learning experiences and reflections from a transdisciplinary urban case study in Enkanini informal settlement, South Africa, Sustain. Sci., 2018, DOI: 10.1007/s11625-018-0606-x

[48] Van Eijk T., Farming Systems Research and Spirituality, PhD dissertation, Wageningen University, Wageningen, The Netherlands, 1998

[49] Van Haaften E.H., Linking Ecology and Culture: Towards a Psychology of Environmental Degradation, PhD dissertation, University of Tilburg, Tilburg, the Netherlands, 2002

[50] Vangala R.N.K., Hiremath B.N., Banerjee A., A Theoretical Framework for Knowledge Management in Indian Agricultural Organizations, In: Proceedings of the 2014 International Conference on Information and Communication Technology for Competitive Strategies (November 2014, Udaipur, Rajasthan, India), 2014, Article 6 
[51] Zylstra M. J., Exploring meaningful nature experience, connectedness with nature, and the revitalization of transformative education for sustainability. PhD dissertation, Stellenbosch University, Stellenbosch, South Africa, 2014

[52] Zylstra M.J., Knight A.T., Esler K.J., Lesley L.L.L.,

Connectedness as a Core Conservation Concern: An Interdisciplinary Review of Theory and a Call for Practice, Springer Sci. Rev., 2014, 2, 119-143
[53] Zylstra M.J., Knight A.T., Esler K.J., Lesley L.L.L., Integrating multiple perspectives on the human-nature relationship: A reply to Fletcher 2017, J. Environ. Educ., 2018, DOI: 10.1080/00958964.2018.1497582 